\title{
ON THE SOLUTIONS OF A STOCHASTIC CONTROL SYSTEM. II*
}

\author{
TYRONE DUNCAN $\dagger$ AND PRAVIN VARAIYA $\ddagger$
}

\begin{abstract}
This paper presents generalizations of the work in [1], [2] to include controlled stochastic processes which take values in a certain class of Fréchet spaces. The crucial result is an extension of Girsanov's technique for defining solutions of stochastic differential equations by an absolutely continuous transformation of measures. The result is used to prove existence results for stochastic control problems and for a class of two-person zero sum games.
\end{abstract}

1. Introduction and summary. Consider a controlled stochastic process represented by the stochastic differential equation

$$
d X(t)=f(t, X, u(t, X)) d t+d B(t), \quad t \in[0,1],
$$

where $B(t)$ is a Fréchet-valued Brownian motion, $X(t)$ is the state process. The drift $f$, and the control $u$, depend at any time $t$ on the past of the state process, $\{X(s), s \leqq t\}$. For the case where the Fréchet space is $R^{n}$, a satisfactory theory dealing with the problem of existence of solutions of the differential equation and existence of optimal control laws is now available [1], [2]. A crucial building block in this theory consists in defining a solution of the differential equation via an absolutely continuous transformation of measures. Each control thereby defines a solution characterized by its (unique) probability law which is absolutely continuous with respect to Wiener measure. Thus the influence of a control law upon the system is captured in the Radon-Nikodym derivative of the resulting probability law with respect to Wiener measure. Questions dealing with the existence of an optimal control can then be converted into questions about the compactness (in an appropriate sense) of the set of Radon-Nikodym derivatives. The measure transformation technique mentioned above is due originally to Girsanov [16].

This paper deals with these same questions for the case where the state space is infinite-dimensional. The problem of characterizing Brownian motion in infinite-dimensional spaces is a difficult one and has been resolved for certain Fréchet spaces only. This is described in the next section, where some additional properties of such Brownian motion as sample continuity and stochastic integration are established also. In $\S 3$ the result of Girsanov is extended to cover the differential equation under consideration. Once this has been achieved the techniques of [1], [2] apply without change and the existence of optimal controls and saddle points follows easily. This is sketched in $\S 4$.

2. Preliminaries for defining Brownian motion. To define probability measures on Fréchet spaces the results of Dudley-Feldman-LeCam [3] are used. The

* Received by the editors September 25, 1973, and in revised form August 29, 1974.

† Department of Applied Mathematics and Statistics, State University of New York at Stony Brook, Stony Brook, New York 11790. This research was supported in part by the National Science Foundation under Grants GK-24151 and GK-32136.

$\ddagger$ Department of Electrical Engineering and Computer Science and Electronics Research Laboratory, University of California, Berkeley, California 94720. This research was supported in part by the National Science Foundation under Grant GK-10656X3. 
latter are generalizations of the work of Gross [4]. While the generality of the immediately following discussion is not used subsequently, it does serve to indicate some directions along which the results reported here can be further pursued. Some notation and definitions are introduced first.

For a locally convex Huasdorff topological (real) vector space $X$ let $X^{*}$ denote its topological dual. A dual system or duality over the reals consists of two vector spaces $X, Y$ and a bilinear form $\langle\cdot, \cdot\rangle: X \times Y \rightarrow R$ that separates points for both $X$ and $Y$. For a pair $X, Y$ in duality, let $F D(X)$ denote the collection of all finite-dimensional subspaces of $X$. For $G \subset X$, let $\mathscr{M}(Y, G)$ be the $\sigma$-algebra of $Y$ generated by the sets $\{y:\langle x, y\rangle \in B\}$, where $x \in G, B$ is a Borel set in $R$. Thus $\mathscr{M}(Y, G)$ is the smallest $\sigma$-algebra on $Y$ for which each $x \in G$, regarded as a function on $Y$, is measurable. Finally let $\mathscr{C}(Y, X)=\bigcup\{\mathscr{M}(Y, G) \mid G \in F D(x)\}$. A cylinder set measure on $Y$ is any nonnegative, finitely additive set function $m$ on $\mathscr{C}(Y, X)$ with $m(Y)=1$ such that $m$ is countably additive on $\mathscr{M}(Y, G)$ for each $G \in F D(X)$.

The following notion of a measurable seminorm given in [3] is crucial to the analysis.

Definition 1. Given a duality $X, Y,\langle\cdot, \cdot\rangle$, and a cylinder set measure $m$ on $Y$, a seminorm $|\cdot|$ on $Y$ is said to be $m$-measurable if for each $\varepsilon>0$ there is a $G \in F D(Y)$ such that if $F \in F D(X)$ and $F \perp G$ (i.e., $\langle x, y\rangle=0$ for $x \in F, y \in G)$, then

$$
m\left\{y:\left|y-F^{\perp}\right| \leqq \varepsilon\right\} \geqq 1-\varepsilon
$$

or equivalently

$$
m^{*}\{y:|y-G| \leqq \varepsilon\} \geqq 1-\varepsilon,
$$

where $m^{*}$ is the outer measure on $\mathscr{C}(Y, X)$ induced by $m$.

The next result follows from [3, Thm. 2].

FundaMentAL THEOREM. Let $|\cdot|$ be a Mackey-continuous seminorm on $Y$ and suppose that it is m-measurable. Then the cylinder set measure induced by $m$ on the Banach space $Y /|\cdot|$ obtained from $Y$ via the seminorm $|\cdot|$ extends to a regular Borel measure.

From here on the discussion is specialized to a fixed, separable Hilbert space $H$. It is assumed that there is given for each $t \in R_{+}$a cylinder set measure $p_{t}$ on $H$ such that $p_{t}$ is a canonical normal distribution on $H$ with variance parameter $t$ (see [4] or [5]). ${ }^{1}$ It is also assumed that there is given an increasing family of Mackey-continuous seminorms $|\cdot|_{j}, j=1,2, \cdots$, on $H$. Let $F$ be the Fréchet space obtained from $H$ with respect to the topology defined by the seminorms $|\cdot|_{j}$ by completion modulo the intersection of their null spaces (which without loss of generality is assumed to equal $\{0\}$ ). As a corollary to the Fundamental Theorem it is proved in $\left[3\right.$, Cor. 2.1] that each $p_{t}, t \in R_{+}$, extends to a regular Borel measure, denoted $\mu_{t}$, on $F$. For future reference note that $H \subset F, F^{*} \subset H^{*}$

${ }^{1}$ This means that if $C \in \mathscr{C}(H, H)$ is of the form $C=P^{-1}(E)$, where $P$ is the orthogonal projection of $H$ onto a subspace $L \in F D(H)$ and $E$ is a Borel subset of $L$, then

$$
p_{t}(C)=(2 \pi t)^{-n / 2} \int_{E} \exp \left(-\frac{1}{2 t}|x|_{H}^{2}\right) d x,
$$

where $n$ is the dimension of $L$, and $|\cdot|_{H}$ denotes the norm on $H$ induced by its inner product. 
$=H$, and define the maps $i: H \rightarrow F, j: F^{*} \rightarrow H^{*}$, as the canonical injections. Finally let $\mathscr{B}(F)$ denote the Borel sets of $F$. Throughout, $H$ and $F$ denote the spaces introduced here.

The collection $\mu_{t}, t \in R_{+}$, will be used now to define a Brownian motion with values in $F$. For each $t \in R_{+}$let $F^{t}$ be a copy of $F$. For each finite collection $t_{1}<\cdots<t_{n}$, let $\mu_{t_{1}, \cdots, t_{n}}$ be the measure on $\left(\prod_{i=1}^{n} F^{t_{i}}, \prod_{i=1}^{n} \mathscr{B}\left(F^{t_{i}}\right)\right)$ defined by

$\int_{\Lambda_{1}} \cdots \int_{\Lambda_{n}} d \mu_{t_{1}, \cdots, t_{n}}=\int_{\Lambda_{1}} \int_{\Lambda_{2}-x_{1}} \cdots \int_{\Lambda_{n}-\Sigma_{1}^{n-1} x_{i}} d \mu_{t_{n-t_{n-1}}}\left(x_{n}\right) \cdots d \mu_{t_{2}-t_{1}}\left(x_{2}\right) d \mathrm{Y}_{t_{1}}\left(x_{1}\right)$

for $\Lambda_{i} \in \mathscr{B}\left(F^{t_{i}}\right), i=1, \cdots, n$. Since sets of the form $\prod_{i=1}^{n} \Lambda_{i}$ generate the product $\sigma$-algebra $\prod_{i=1}^{n} \mathscr{B}\left(F^{t_{i}}\right)$ the measure $\mu_{t_{1}, \cdots, t_{n}}$ is defined. To show that the family of measures $\mu_{t_{1}, \cdots, t_{n}}$ is a projective system, it is necessary to verify consistency. Since $F$ is separable in the topology determined by the countable seminorms $|\cdot|_{j}$, the measure $\mu_{t_{1}, \cdots, t_{n}}$ is determined by sets of the form $\Lambda_{i}=(I-P) F \oplus \Gamma_{i}$, where $P$ is an orthogonal projection with finite dimensional range and $\Gamma_{i}$ is a Borel subset of $P F$. Let $\Lambda_{1}, \cdots, \Lambda_{n}$ be such sets, and suppose $\Lambda_{j}=F$. Then

$$
\begin{aligned}
\int_{\Lambda_{1}} \cdots \int_{\Lambda_{n}} d \mu_{t_{1}, \cdots, t_{n}} & =\int_{\Lambda_{1}} \int_{\Lambda_{2}-x_{1}} \cdots \int_{\Lambda_{n}-\Sigma_{1}^{n-1} x_{i}} d \mu_{t_{n}-t_{n-1}}\left(x_{n}\right) \cdots d \mu_{t_{1}}\left(x_{1}\right) \\
& =\int_{\Gamma_{1}} \int_{\Gamma_{2}-x_{1}} \cdots \int_{\Gamma_{n}-\Sigma_{1}^{n-1} x_{i}} d p_{t_{n}-t_{n-1}}\left(x_{n}\right) \cdots d p_{t_{1}}\left(x_{1}\right) \\
& =\int_{\Gamma_{1}} \cdots \int_{\Gamma_{j-1}-\Sigma_{1}^{j-2} x_{i}} \int_{\Gamma_{j+1}-\Sigma_{1}^{j} x_{i}} \cdots \int_{\Gamma_{n}-\Sigma_{1}^{n-1} x_{i}} d p_{t_{n}-t_{n-1}}\left(x_{n}\right) \\
& =\int_{\Lambda_{1}} \cdots \int_{\Lambda_{j-1}} \int_{\Lambda_{j+1}} \cdots \int_{\Lambda_{n}} d \mu_{t_{1}, \cdots, t_{j-1}, t_{j+1}, \cdots, t_{n}} .
\end{aligned}
$$

In the above $p$ is the measure of a Brownian motion with values in the finitedimensional space $P F$ and so the third equality above follows from the Markov property of such a process. Since each of the measures $\mu_{t}, t \in R_{+}$, is a regular Borel measure, the projective system of measures admits a projective limit $[6$, p. 49]. The projective limit thus obtained is denoted $(\Omega, \mathscr{F}, P)$. Evidently, $(\Omega, \mathscr{F})=\prod_{t \in R_{+}}\left(F^{t}, \mathscr{B}\left(F^{t}\right)\right)$. It will be assumed that $(\Omega, \mathscr{F}, P)$ is complete. For each $t$ let $X_{t}$ denote the $F$-valued evaluation map on $\Omega$ at $t$. Let $\mathscr{F}_{t}$ be the smallest completed $\sigma$-algebra with respect to which $X_{s}$ is measurable for $s \leqq t$. Then $\left(X_{t}, \mathscr{F}_{t}, P\right)_{t \in R_{+}}$is a Brownian motion as defined below.

Definition 2. A stochastic process $\left(X_{t}, \mathscr{F}_{t}, P\right)_{t \in R_{+}}$(or simply $\left(X_{t}, P\right)_{t \in R_{+}}$or $\left(X_{t}\right)$ if there is no ambiguity) is said to be a Brownian motion with values in the Fréchet space $F$ induced from a family of canonical normal distributions on a Hilbert space $H$, if for each $l \in F^{*}$ (the topological dual of $F$ ) the real-valued process $\left(\left\langle l, X_{t}\right\rangle, \mathscr{F}_{t}, P\right)_{t \in R_{+}}$is a real-valued Brownian motion with $E\left\langle l, X_{t}\right\rangle^{2}$ $=t|j|_{H}^{2}$. (Here and throughout $|\cdot|_{H}$ denotes the norm on $H$ and $j: F^{*} \rightarrow H^{*}$ is the canonical injection mentioned before.)

The process $\left(Y_{t}, P\right)_{t \in R_{+}}$is said to be a modification of the process $\left(X_{t}, P\right)_{t \in R_{+}}$ if for each $t \in R_{+} X_{t}(\omega)=Y_{t}(\omega)$ a.s. (the null set $\left\{X_{t} \neq Y_{t}\right\}$ may depend on $t$ ). 
For Fréchet-valued Brownian motion there is a modification which has continuous sample paths as shown by the following lemma.

LeMma 1. Let $\left(X_{t}, \mathscr{F}_{t}, P\right)_{t \in R_{+}}$be a Fréchet-valued Brownian motion. There is a modification of $X_{t}$ with continuous sample paths.

Proof. Since a countable number of seminorms determine the topology of a Fréchet space it suffices to verify the continuity of the sample function of Brownian motion with respect to each of these countable number of seminorms and therefore it is enough to prove the lemma for a Banach-valued Brownian motion.

Fernique [7] has shown that for a Gaussian random variable $X$ on a topological vector space with a measurable seminorm $|\cdot|$ there is an $\alpha>0$ such that

$$
E \exp \alpha|X|^{2}<\infty
$$

Combining (1) with a result of Nelson [8, Thm. 2] as used by Gross [9, p. 134] it follows that the Banach valued Brownian motion has a modification with continuous sample paths.

Since stochastic integrals will be used subsequently, a family of processes has to be described that can serve as integrands. The following definition gives such a family.

DeFINITION 3. Let $\tilde{H}$ be a separable Hilbert space. An $\tilde{H}$-valued stochastic process $\left(\psi_{t}\right)_{t \in R_{+}}$on $(\Omega, \mathscr{F}, P)$ that is adapted to $\left(\mathscr{F}_{t}\right)_{t \in R_{+}}$is said to be predictable if the map $\psi_{t}(\omega): R_{+} \times \Omega \rightarrow \tilde{H}$ is measurable with respect to the $\sigma$-algebra on $R_{+} \times \Omega$ generated by the left-continuous $\tilde{H}$-valued processes adapted to $\left(\mathscr{F}_{t}\right)_{t \in R_{+}}$.

Real-valued stochastic integrals will now be defined from $F$-valued Brownian motion and predictable $H$-valued processes.

LEMMA 2. Let $\left(\psi_{t}\right)_{t \in R_{+}}$be a predictable H-valued process with

$$
E \int_{0}^{\infty}\left|\psi_{t}\right|_{H}^{2} d t<\infty,
$$

and let $\left(B_{t}, \mathscr{F}_{t}, P\right)_{t \in R_{+}}$be an $F$-valued Brownian motion. Then the real-valued process $\left(Y_{t}, \mathscr{F}_{t}, P\right)_{t \in R_{+}}$,

$$
Y_{t}=\int_{0}^{t}\left\langle\psi_{s}, d B_{s}\right\rangle,
$$

is a square integrable martingale which has a modification with continuous sample paths. (The integral (3) is defined in the course of the proof.)

Proof. Let $\varepsilon_{n}>0$ be a sequence decreasing to zero and for each $n$ let $P_{\varepsilon_{n}}$ be a projection on $H$ with finite-dimensional range $P_{\varepsilon_{n}} H \subset j F^{*}$ such that

$$
E \int_{0}^{\infty}\left|\left(I-P_{\varepsilon_{n}}\right) \psi_{t}\right|_{H}^{2}<\varepsilon_{n} .
$$

Let $l_{1}, \cdots, l_{k}$ be an orthonormal basis for $P_{\varepsilon_{n}} H$. The process $Y_{t}\left(P_{\varepsilon_{n}}\right)$ defined by

$$
\begin{aligned}
Y_{t}\left(P_{\varepsilon_{n}}\right) & =\int_{0}^{t}\left\langle P_{\varepsilon_{n}} \psi_{s}, d B_{s}\right\rangle \\
& =\int_{0}^{t} \sum_{i=1}^{k}\left\langle l_{i}, \psi_{s}\right\rangle d\left\langle l_{i}, B_{s}\right\rangle
\end{aligned}
$$


is a (continuous) martingale since by the definition of the process $\left(B_{t}\right),\left(\left\langle l_{i}, B_{t}\right\rangle\right)$ is a real-valued Brownian motion. By Doob's inequality [10, p. 353] the sequence of continuous martingales $\left(Y_{t}\left(P_{\varepsilon_{n}}\right)\right)$ converges uniformly on compact subsets of $R_{+}$because

$$
E\left|Y_{t}\left(P_{\varepsilon_{n}}\right)-Y_{t}\left(P_{\varepsilon_{m}}\right)\right|^{2}=E \int_{0}^{t}\left|\left(P_{\varepsilon_{n}}-P_{\varepsilon_{m}}\right) \psi_{s}\right|^{2} d s
$$

which vanishes as $m, n$ increase to infinity. The integral (3) is defined as the limit of the martingales $Y_{t}\left(P_{\varepsilon_{n}}\right)$. Clearly the limit does not depend on the particular choice of the projections $P_{\varepsilon_{n}}$ and $Y_{t}$ is evidently square integrable.

COROLlaRY 1. Let $\left(\psi_{t}\right)_{t \in R_{+}}$be a predictable H-valued process with

$$
\int_{0}^{t}\left|\psi_{s}\right|_{H}^{2} d s<\infty \quad \text { a.s. for all } t .
$$

Then the real-valued process $Z_{t}$ defined by

$$
Z_{t}=\int_{0}^{t}\left\langle\psi_{s}, d B_{s}\right\rangle
$$

is a locally square integrable martingale which has a modification with continuous sample paths. ${ }^{2}$

The following representation of square integrable functionals on the $F$-valued Brownian motion probability space will be useful subsequently. For $R^{n}$-valued Brownian motion, K. Itô [11] has obtained this representation by describing results of Wiener [12] and Cameron-Martin [13] in terms of stochastic integrals.

Proposition 1. Let $(\Omega, \mathscr{F}, P)$ be the probability space for an $F$-valued Brownian motion $\left(B_{t}, \mathscr{F}_{t}\right)_{t \in R_{+}}$. Let $f$ be a real-valued square integrable functional on $(\Omega, \mathscr{F}, P)$. Then $f$ can be represented as

$$
f=c+\int_{0}^{\infty}\left\langle\psi_{s}, d B_{s}\right\rangle
$$

where $c=E f$ and $\left(\psi_{t}\right)_{t \in R_{+}}$is a predictable $H$-valued process with

$$
E \int_{0}^{\infty}\left|\psi_{t}\right|_{H}^{2} d t<\infty
$$

Proof. Since $H$ is separable so is $F$, hence by the Hahn-Banach theorem, there is a countable family $\Gamma \subset F^{*}$ that separates points of $F$. Consider the random variables $\left\langle\gamma, \int_{0}^{t} d B_{s}\right\rangle$, where $\gamma \in \Gamma, t \in R_{+}$and let $\mathscr{A}$ be the algebra of real-valued random variables formed from these and the constant random variables. Since the random variables $\left\langle\gamma, \int_{0}^{t} d B_{s}\right\rangle$ are jointly Gaussian, it follows that $\mathscr{A} \subset L^{2}(P)$ and furthermore

$$
\exp \left(\sum_{i=1}^{n} a_{i}\left|f_{i}\right|\right) \in L^{2}(P)
$$

\footnotetext{
${ }^{2} m_{t}$ is said to be a locally square integrable martingale if there exists a sequence of stopping times $T_{n} \uparrow \infty$ a.s. such that $m_{t \wedge T_{n}}$ is a square integrable martingale for each $n$.
} 
for $a_{i} \in R$ and $f_{i} \in \mathscr{A}$. Since $F$ is separable, the family of random variables $\left\langle\gamma, \int_{0}^{t} d B_{s}\right\rangle, \gamma \in \Gamma, t \in R_{+}$, generates the $\sigma$-algebra $\mathscr{F}$. By a result of Segal [14, Lemma 2.1] it follows that $\mathscr{A}$ is dense in $L^{2}(P)$.

Let $f \in L^{2}(P)$. There exists then a sequence $g_{n}$ in $\mathscr{A}$ such that $E\left|f-g_{n}\right|^{2} \rightarrow 0$. By properties of finite-dimensional Brownian motion, $g_{n}$ has a representation of the form (7) (see [15]), i.e.,

$$
g_{n}=c_{n}+\int_{0}^{\infty}\left\langle\psi_{s}^{n}, d B_{s}\right\rangle
$$

where $c_{n}=E g_{n}$ and $\left(\psi_{t}^{n}\right)$ is a predictable process with values in some finitedimensional subspace $L$ of $H$ and with $L \subset j F^{*}$. Since $L^{2}(P)$-convergence implies $L^{1}(P)$-convergence it follows that $c_{n}$ converges to $c$. Furthermore the stochastic integrals in (8) must be Cauchy; hence

$$
\int_{0}^{\infty} E\left|\psi_{t}^{n}-\psi_{t}^{m}\right|_{H}^{2} d t \rightarrow 0
$$

as $m, n \rightarrow \infty$. Since the sequence of processes $\left(\psi_{t}^{m}\right)$ are predictable, there must exist a predictable process $\left(\psi_{t}\right)$ such that

$$
\int_{0}^{\infty}\left|\psi_{t}^{n}-\psi_{t}\right|_{H}^{2} \rightarrow 0
$$

and evidently (7) is satisfied.

3. Transformation of measures. Theorem 1 below describes how Fréchetvalued Brownian motion is transformed by changing the probability measure by an absolutely continuous substitution of the measure. This result was first established by Girsanov [16] for the case of $R^{n}$-valued Brownian motion. The result has been presented in [17] and a related result is given in [18].

THEOREM-1. Let $\left(B_{t}, \mathscr{F}_{t}, P\right)_{t \in[0,1]}$ be an F-valued Brownian motion and let $\left(\psi_{t}\right)_{t \in[0,1]}$ be a predictable $H$-valued process such that

$$
\int_{0}^{1}\left|\psi_{t}\right|_{H}^{2} d t<\infty \quad \text { a.s. } P \text {. }
$$

Define the nonnegative process $\left(M_{t}, \mathscr{F}_{t}, P\right)_{t \in[0,1]} b y$

$$
M_{t}=\exp \left[\int_{0}^{t}\left\langle\psi_{s}, d B_{s}\right\rangle-\frac{1}{2} \int_{0}^{t}\left|\psi_{s}\right|_{H}^{2} d s\right] .
$$

Then

$$
E\left(M_{1}\right) \leqq 1
$$

Suppose that

$$
E\left(M_{1}\right)=1
$$

then the process $\left(\widetilde{B}_{t}, \mathscr{F}_{t}, \widetilde{P}\right)_{t \in[0,1]}$ is an F-valued Brownian motion where

$$
\widetilde{B}_{t}=B_{t}-\int_{0}^{t} i \psi_{s} d s
$$


and the probability measure $\widetilde{\boldsymbol{P}}$ is given by

$$
\frac{d \widetilde{P}}{d P}=M_{1} .
$$

Proof. Define the increasing sequence of stopping times $T_{n}$ by

$$
T_{n}=\left\{\begin{array}{l}
\inf \left\{t \mid M_{t}>n\right\}, \\
1 \text { if the set above is empty }
\end{array}\right.
$$

Because of (9) $M_{t}$ has (a modification with) continuous sample paths, so $T_{n} \uparrow 1$ a.s. $P$, and in particular,

$$
\lim _{n \rightarrow \infty} M_{t \wedge T_{n}}=M_{t} \text { a.s. } P \text {. }
$$

By the main result in [19], the processes $\left(M_{t}\right)$ and $\left(M_{t \wedge T_{n}}\right)$ satisfy

$$
\begin{aligned}
& M_{t}=1+\int_{0}^{t} M_{s}\left\langle\psi_{s}, d B_{s}\right\rangle \\
& M_{t \wedge T_{n}}=1+\int_{0}^{t \wedge T_{n}} M_{s}\left\langle\psi_{s}, d B_{s}\right\rangle .
\end{aligned}
$$

Since $\left(M_{t \wedge T_{n}}\right)$ is bounded, (15) implies that it is a martingale and hence

$$
E\left(M_{t \wedge T_{n}}\right)=E\left(M_{T_{n}}\right)=1 .
$$

An application of Fatou's lemma to this result and (13) yields (11).

From now on suppose that (12) holds, so that from (14) it follows that $\left(M_{t}, \mathscr{F}_{t}, P\right)_{t \in[0,1]}$ is in fact a martingale. Let $l \in H$ be fixed and consider the process $\left(N_{t}, \mathscr{F}_{t}, \widetilde{P}\right)_{t \in[0,1]}$, where

$$
N_{t}=\left\langle l, \widetilde{B}_{t}\right\rangle=\left\langle l, B_{t}\right\rangle-\int_{0}^{t}\left\langle l, \psi_{s}\right\rangle d s .
$$

The theorem will be proved once it is shown that

$$
\begin{aligned}
& \left(N_{t}, \mathscr{F}_{t}, \widetilde{P}\right) \quad \text { is a martingale, } \\
& \tilde{E}\left(N_{t}^{2}-N_{s}^{2} \mid \mathscr{F}_{s}\right)=|l|_{H}^{2}(t-s),
\end{aligned}
$$

where $\widetilde{E}$ denotes expectation with respect to the measure $\widetilde{P}$. Now to prove (16) it is sufficient to show instead that

$$
\left(M_{t} N_{t}, \mathscr{F}_{t}, P\right) \text { is a martingale. }
$$

Because suppose that $(18)$ is true. Then using the fact that $\left(M_{t}, P\right)$ is a martingale,

$$
\begin{aligned}
\tilde{E}\left(N_{t} \mid \mathscr{F}_{s}\right) & =\frac{E\left(M_{t} N_{t} \mid \mathscr{F}_{s}\right)}{E\left(M_{t} \mid \mathscr{F}_{s}\right)} \quad(\text { by }[20, \mathrm{p} .345]) \\
& =\frac{M_{s} N_{s}}{M_{s}} \quad(\text { by }(18)) \\
& =N_{s},
\end{aligned}
$$


which is equivalent to (16). Now

$$
M_{t} N_{t}=M_{t}\left\langle l, B_{t}\right\rangle-M_{t} \int_{0}^{t}\left\langle l, \psi_{s}\right\rangle d s .
$$

Applying the differentiation formula for continuous martingales [15] gives

$$
\begin{aligned}
M_{t} N_{t}-M_{0} N_{0}= & \int_{0}^{t} M_{s}\left\langle l, d B_{s}\right\rangle-\int_{0}^{t} M_{s}\left\langle l, \psi_{s}\right\rangle d s \\
& +\int_{0}^{t} N_{s} d M_{s}+\int_{0}^{t} M_{s}\left\langle l, \psi_{s}\right\rangle d s \\
= & \int_{0}^{t} M_{s}\left\langle l, d B_{s}\right\rangle+\int_{0}^{t} N_{s} d M_{s},
\end{aligned}
$$

which clearly implies (18).

It remains to prove (17). To this end note that

$$
\tilde{E}\left(N_{t}^{2}-N_{s}^{2} \mid \mathscr{F}_{s}\right)=\frac{E\left(M_{t}\left(N_{t}^{2}-N_{s}^{2}\right) \mid \mathscr{F}_{s}\right)}{M_{s}},
$$

and apply the differentiation formula to $M_{t} N_{t}^{2}$ to obtain

$$
\begin{aligned}
M_{t} N_{t}^{2}-M_{0} N_{0}^{2}= & \int_{0}^{t} N_{s}^{2} d M_{s}+2 \int_{0}^{t} M_{s} N_{s}\left\langle l, d B_{s}\right\rangle-2 \int_{0}^{t} M_{s} N_{s}\left\langle l, \psi_{s}\right\rangle d s \\
& +2 \int_{0}^{t} N_{s} M_{s}\left\langle l, \psi_{s}\right\rangle d s+\left.\int_{0}^{t} M_{s} l l\right|_{H} ^{2} d s .
\end{aligned}
$$

Substitution of this into (19) gives

$$
\tilde{E}\left(N_{t}^{2}-N_{s}^{2} \mid \mathscr{F}_{s}\right)=\frac{E\left(\int_{s}^{t} M_{\tau}|l|_{H}^{2} d \tau \mid \mathscr{F}_{s}\right)}{M_{s}}=|l|_{H}^{2}(t-s),
$$

and so (17) is proved.

In many applications of the transformation of measures technique of Theorem 1 the crucial difficulty is to verify that $\widetilde{P}(\Omega)=1$. The next result gives a sufficient condition for $\widetilde{P}(\Omega)=1$. It is due to $\mathrm{V}$. Beneš.

Lemma 3 (Beneš). Let $\left(B_{t}, \mathscr{F}_{t}, P\right)_{t \in[0,1]}$ be an F-valued Brownian motion. Let $\left(\psi_{t}\right)_{t \in[0,1]}$ be a predictable $H$-valued process such that

$$
|\psi(t, B)|_{H} \leqq K+K^{\prime} \sup _{s \in[0, t]}\left|B_{s}\right|,
$$

where $|\cdot|$ is a seminorm on $F$ and $K, K^{\prime}$ are constants. Then there is $\alpha>1$ and $M<\infty$, depending only on $K$ and $K^{\prime}$, such that

$$
E \exp \alpha\left[\int_{0}^{1}\left\langle\psi_{s}, d B_{s}\right\rangle-\frac{1}{2} \int_{0}^{1}\left|\psi_{s}\right|_{H}^{2} d s\right]<M .
$$

In particular,

$$
E \exp \left[\int_{0}^{1}\left\langle\psi_{s}, d B_{s}\right\rangle-\frac{1}{2} \int_{0}^{1}\left|\psi_{s}\right|_{H}^{2} d s\right]=1
$$


Proof. For any $\left(\psi_{t}\right)$ denote

$$
\zeta(\psi)=\int_{0}^{1}\left\langle\psi_{s}, d B_{s}\right\rangle-\frac{1}{2} \int_{0}^{1}\left|\psi_{s}\right|_{H}^{2} d s .
$$

Fix $\left(\psi_{t}\right)$ satisfying $(20)$ and let

$$
T_{n}=\inf \left\{\left.t\left|\int_{0}^{t}\right| \psi_{s}\right|_{H} ^{2} d s>n\right\} .
$$

Evidently $T_{n} \uparrow 1$ a.s. and let $\psi_{t}^{n}=\psi_{t \wedge T_{n}}$. Let $\alpha>1$. Let $\widetilde{B}_{t}^{n}=B_{t}-\alpha \int_{0}^{t} i \psi_{s}^{n} d s$. Since $\int_{0}^{1}\left|\psi_{t}^{n}\right|_{H}^{2} d t \leqq n$, it is easy to use the proof of [16, Lemma 1] to show that $E \exp \zeta\left(\alpha \psi^{n}\right)=1$. Hence by Theorem $1,\left(\widetilde{B}_{t}^{n}, \mathscr{F}_{t}, \widetilde{P}^{n}\right)_{t \in[0,1]}$ is a Brownian motion, where $d \widetilde{P}^{n}=\left[\exp \zeta\left(\alpha \psi^{n}\right)\right] d P$. Now

$$
\left|\psi_{t}^{n}\right|_{H} \leqq K+K^{\prime} \sup _{s \in[0, t]}\left|B_{s}\right|
$$

so that

$$
\begin{gathered}
\left|B_{t}\right| \leqq\left|\widetilde{B}_{t}^{n}\right|+\alpha \int_{0}^{t}\left[K+K^{\prime} \sup _{u \in[0, s]}\left|B_{u}\right|\right] d s, \\
\sup _{s \in[0, t]}\left|B_{s}\right| \leqq \sup _{s \in[0, t]}\left|\widetilde{B}_{s}^{n}\right|+\alpha K t+\alpha K^{\prime} \int_{0}^{t} \sup _{u \in[0, s]}\left|B_{u}\right| d s,
\end{gathered}
$$

and hence by Gronwall's inequality,

$$
\|B\| \leqq\left(\alpha K+\left\|\widetilde{B}^{n}\right\|\right) \exp \alpha K^{\prime},
$$

where $\|X\|=\sup _{s \in[0,1]}\left|X_{s}\right|$. From $(20),\left|\psi_{s}^{n}\right|^{2} \leqq 2 K^{2}+2 K^{\prime}\|B\|^{2}$, so that

$$
\int_{0}^{1}\left|\psi_{s}^{n}\right|_{H}^{2} d s \leqq 2 K^{2}+4 K^{\prime}\left(\alpha^{2} K^{2}+\left\|\tilde{B}^{n}\right\|^{2}\right) \exp 2 \alpha K^{\prime} .
$$

By the differentiation formula,

$$
\begin{aligned}
E \exp \alpha \zeta\left(\psi^{n}\right) & =E \exp \left[\zeta\left(\alpha \psi^{n}\right)+\frac{\alpha^{2}-\alpha}{2} \int_{0}^{1}\left|\psi_{s}\right|_{H}^{2} d s\right] \\
& \leqq E \exp \zeta\left(\alpha \psi^{n}\right) \exp \left(\frac{\alpha^{2}-\alpha}{2}\right)\left[2 K^{2}+4 K^{\prime}\left(\alpha^{2} K^{2}+\left\|\widetilde{B}^{n}\right\|^{2}\right) \exp 2 \alpha K^{\prime}\right] \\
& =N E^{n} \exp \left[\left(\frac{\alpha^{2}-\alpha}{2}\right)\left(4 K^{\prime} \exp 2 \alpha K^{\prime}\right)\left\|\widetilde{B}^{n}\right\|^{2}\right],
\end{aligned}
$$

where $N$ is a constant and $E^{n}$ denotes expectation with respect to $\tilde{P}^{n}$. Since $\left(\widetilde{B}^{n}, \widetilde{P}^{n}\right)$ is a Brownian motion by a result of Fernique [7] there exists $\gamma>0$ such that

$$
E^{n} \exp \gamma\left\|\widetilde{B}^{n}\right\|^{2}=A<\infty
$$

independent of $n$. Let $\alpha>1$ be so small that $\left(\left(\alpha^{2}-\alpha\right) / 2\right)\left(4 K^{\prime} \exp 2 \alpha K^{\prime}\right)<\gamma$. Then

$$
E \exp \alpha \zeta\left(\psi^{n}\right)<N A,
$$


and so (21) follows by Fatou's lemma. The final assertion is then immediate because (21) implies that $\exp \left[\int_{0}^{t}\left\langle\psi_{s}, d B_{s}\right\rangle-\frac{1}{2} \int_{0}^{t}\left|\psi_{s}\right|_{H}^{2} d s\right]$ is a martingale.

4. Preliminaries for optimization. The system to be controlled is represented by the stochastic differential equation

$$
d X(t)=i f(t, X, u(t, X)) d t+d B(t), \quad t \in[0,1],
$$

where $B_{t}$ is an $F$-valued Brownian motion, $X_{t} \in F$ is the state with $X_{0}=0$ a.s., and $u$ is the control law taking values in a prespecified compact subset $U \subset F$ called the control set. The function $f$ takes values in $H$ and $i: H \rightarrow F$ is the canonical injection. The first difficulty to be resolved is to define the solution of the differential equation (22) for a large class of control laws. This is achieved in the following manner. One starts with a process $\left(X_{t}, \mathscr{F}_{t}, P_{0}\right)_{t \in[0,1]}$ which is an $F$-valued Brownian motion. For a given control law $u$ an $F$-valued process $B_{t}^{u}$ is defined by

$$
B_{t}^{u}=X_{t}-\int_{0}^{t} i f(s, X, u(s, X)) d s .
$$

Next the probability measure $P_{0}$ is replaced by another probability measure $P^{u}$ such that the process $\left(B_{t}^{u}, \mathscr{F}_{t}, P^{u}\right)_{t \in[0,1]}$ is an $F$-valued Brownian motion. The process $\left(X_{t}, \mathscr{F}_{t}, P^{u}\right)_{t \in[0,1]}$ is then regarded as the solution of (22) corresponding to the control law $u$. To make this procedure precise the following notations and definitions are useful.

Definition 4. (a) $\mathscr{C}$ is the linear space of all $F$-valued continuous functions, denoted by $z$, on $[0,1]$.

(b) For $t \in[0,1], \mathscr{S}_{t}$ is the smallest $\sigma$-algebra of subsets of $\mathscr{C}$ which contain all sets of the form $\{z \in \mathscr{C} \mid z(s) \in A\}$, where $s \in[0, t]$ and $A$ is a (topological) Borel subset of $F . \mathscr{S}=\mathscr{S}_{1}$.

Throughout the remainder of this paper $\Omega$ is a fixed probability space with an increasing family of $\sigma$-algebras $\mathscr{F}_{t}, t \in[0,1] . \mathscr{F}=\mathscr{F}_{1}$. It will be necessary to consider different probability measures on the space $(\Omega, \mathscr{F})$. If $Y_{t}$ is a family of measurable functions on $\left(\Omega, \mathscr{F}_{t}\right)$ and if $P$ is a probability measure on $(\Omega, \mathscr{F})$, the stochastic process corresponding to $P$ and the family $Y_{t}$ will be denoted by $\left(Y_{t}, \mathscr{F}_{t}, P\right)_{t \in[0,1]}$. Then the same family $Y_{t}$ generates different stochastic processes corresponding to different probability measures. Finally let $P_{0}$ be a distinguished probability measure, and let $X_{t}$ be a distinguished family of $F$-valued measurable functions on $\left(\Omega, \mathscr{F}_{t}\right), t \in[0,1]$, such that the process $\left(X_{t}, \mathscr{F}_{t}, P_{0}\right)_{t \in[0,1]}$ is an $F$ valued Brownian motion with continuous sample paths. Unless mentioned otherwise the process $X_{t}$ refers to this process. Also $E_{0}$ will denote expectation with respect to $P_{0}$. The measure induced on $(\mathscr{C}, \mathscr{S})$ by the process $X_{t}$ is denoted by $\mu$ and will be called the Wiener measure on $(\mathscr{C}, \mathscr{S})$.

The following conditions are imposed on the function $f$ in (22).

f1. $f$ is a map from $[0,1] \times \mathscr{C} \times U$ into $H$ and $f$ is measurable with respect to the product $\sigma$-algebra $\mathscr{B} \otimes \mathscr{S} \otimes \mathscr{B}_{u}$, where $\mathscr{B}\left(\mathscr{B}_{u}\right)$ is the family of Borel subsets of $[0,1](U)$.

f2. For $t \in[0,1], f(t, \cdot, \cdot)$ is $\mathscr{S}_{t} \otimes \mathscr{B}_{u}$-measurable.

f3. For $(t, z) \in[0,1] \times \mathscr{C}, f(t, z, \cdot): U \rightarrow H$ is continuous.

f4. There is an increasing function $f_{0}: R_{+} \rightarrow R_{+}$, and a seminorm $|\cdot|$ on $F$ 
such that for $(t, z, u) \in[0,1] \times \mathscr{C} \times U$,

$$
|f(t, z, u)|_{H} \leqq f_{0}(\|z\|),
$$

where $\|z\|=\max _{t \in[0,1]}|z(t)|$. Throughout the remainder the symbols $|\cdot|$ and $\|\cdot\|$ will denote the seminorms assumed here.

f5. For $(t, z) \in[0,1] \times \mathscr{C}, f(t, z, U)=\{f(t, z, u) \mid u \in U\}$ is a closed and convex subset of $H$.

Definition 5. (a) An admissible control (law) is a map $u:[0,1] \times \mathscr{C} \rightarrow U$ which is $\mathscr{B} \otimes \mathscr{S}$-measurable and, further, is such that for each $t \in[0,1], u(t, \cdot)$ is $\mathscr{S}_{t}$-measurable. $\mathscr{U}$ denotes the set of all admissible controls.

(b) The drift corresponding to $u \in \mathscr{U}$ is the function $g_{u}$ given by $g_{u}$ given by $g_{u}(t, z)=f(t, z, u(t, z)) . \mathscr{Y}=\left\{g_{u} \mid u \in U\right\}$.

(c) For $g \in \mathscr{Y}$ and positive integer $n, g^{n}$ is the function given by

$$
g^{n}(t, z)= \begin{cases}g(t, z) & \text { if }|z(s)| \leqq n \text { for } s \in[0, t], \\ 0 & \text { otherwise. }\end{cases}
$$

Definition 6. A function $\phi:[0,1] \times \mathscr{C} \rightarrow H$ is causal if it is $\mathscr{B} \otimes \mathscr{S}$ measurable and if $\phi(t, \cdot)$ is $\mathscr{S}_{t}$-measurable for $t \in[0,1]$.

Definition 7. $\Phi$ is the collection of all causal functions such that $|\phi(t, z)|_{H}$ $\leqq f_{0}(\|z\|)$ for $(t, z) \in[0,1] \times \mathscr{C} . \Phi^{n}=\left\{\left.\phi \in \Phi|| \phi(t, z)\right|_{H} \leqq n\right.$ for all $\left.(t, z)\right\}$.

The next result which follows immediately from [21, Lemma 1$]$ gives a very useful characterization of $\mathscr{Y}$.

Lemma 4. A causal function $g$ is in $\mathscr{Y}$ if and only if $g(t, z) \in f(t, z, U)$ for all $t, z$.

Definition 8. Let $\phi$ be a causal function such that

$$
\int_{0}^{1}|\phi(t, z)|_{H}^{2} d t<\infty \quad \text { for all } z \in \mathscr{C} .
$$

Then $\left.\left(\zeta_{t}(\phi), \mathscr{F}_{t}, P_{0}\right)\right)_{t \in[0,1]}$ denotes the continuous real-valued process defined by

$$
\zeta_{t}(\phi)=\int_{0}^{t}\left\langle\phi(s, X), d X_{s}\right\rangle-\frac{1}{2} \int_{0}^{t}|\phi(s, X)|_{H}^{2} d s .
$$

Let $\zeta(\phi)=\zeta_{1}(\phi)$. Note that (23) is always satisfied for $\phi \in \Phi$.

The problem of the existence of solutions of (22) is resolved in the following result which follows immediately from Theorem 1 .

THEOREM 2. Let $u \in \mathscr{U}$ be such that

$$
E_{0} \exp \zeta\left(g_{u}\right)=1,
$$

where $E_{0}$ denotes expectation with respect to $P_{0}$. Define the probability measure $P_{u} b y$

$$
d P_{u}=\exp \zeta\left(g_{u}\right) d P_{0}
$$

Then the process $\left(B_{t}, \mathscr{F}_{t}, P_{u}\right)_{t \in[0,1]}$ defined by

$$
B_{t}=X_{t}-\int_{0}^{t} i f(s, X, u(s, X)) d s
$$

is an F-valued Brownian motion. 
The next result shows that (24) must be satisfied by every solution of (22).

LEMMA 5. Let $\phi \in \Phi$. Let $\left(Y_{t}, \mathscr{F}_{t}, P\right)_{t \in[0,1]}$ be any process with continuous sample paths such that the stochastic process $\left(B_{t}, \mathscr{F}_{t}, P\right)_{t \in[0,1]}$ defined by

$$
d B_{t}=d Y_{t}-i \phi(t, Y) d t
$$

is an F-valued Brownian motion. Then

$$
\int_{\Omega} \exp \left[-\int_{0}^{1}\left\langle\phi(s, Y), d B_{s}\right\rangle-\frac{1}{2} \int_{0}^{1}|\phi(s, Y)|_{H}^{2} d s\right] d P=1 .
$$

Proof. Define the function $\phi_{n}:[0,1] \times \mathscr{C} \rightarrow H$ by

$$
\phi_{n}(t, z)= \begin{cases}\phi(t, z) & \text { if }|z(s)| \leqq n \text { for } s \in[0, t] \\ 0 & \text { otherwise }\end{cases}
$$

From the definition of $\Phi$ it follows that

$$
\left|\phi_{n}(t, Y(\omega))\right|_{H} \leqq f_{0}(n) \quad \text { for } t \in[0,1], \quad \omega \in \Omega .
$$

By Lemma 3,

$$
\int_{\Omega} \exp \zeta\left(-\phi_{n}\right) d P=1
$$

where

$$
\zeta\left(-\phi_{n}\right)=-\int_{0}^{1}\left\langle\phi_{n}(s, Y), d B_{s}\right\rangle-\frac{1}{2} \int_{0}^{1}\left|\phi_{n}(s, Y)\right|_{H}^{2} d s .
$$

By Theorem 1 the process $\left(Y_{n}(t), \mathscr{F}_{t}, \widetilde{P}_{n}\right)_{t \in[0,1]}$, where

$$
\begin{aligned}
& Y_{n}(t)=\int_{0}^{t} i \phi_{n}(s, Y) d s+B(t), \\
& d \widetilde{P}_{n}=\exp \zeta\left(-\phi_{n}\right) d P,
\end{aligned}
$$

is a Brownian motion with continuous sample paths and hence induces Wiener measure $\mu$ on $(\mathscr{C}, \mathscr{S})$. Let $\varepsilon>0$, and $n$ be so large that

$$
\widetilde{P}_{n}\left(\Omega_{n}\right)=\widetilde{P}_{n}\left(\left\|Y_{n}\right\|<n\right)=\mu(z \in \mathscr{C} \mid\|z\|<n) \geqq 1-\varepsilon .
$$

Now it is clear that $\left\|Y_{n}(\omega)\right\|<n$ only if $\|Y(\omega)\|<n$ and hence

$$
Y_{n}(\omega, t)=Y(\omega, t), \quad \phi_{n}(\omega, t)=\phi(\omega, t) \quad \text { for } \omega \in \Omega_{n}, \quad t \in[0,1],
$$

so that

$$
\widetilde{P}_{n}\left(\Omega_{n}\right)=\int_{\Omega_{n}} \exp \zeta\left(-\phi_{n}\right) d P=\int_{\Omega_{n}} \exp \zeta(-\phi) d P \geqq 1-\varepsilon .
$$

Since $\varepsilon>0$ is arbitrary, the result follows.

COROLlaRY 2. Let $\phi \in \Phi$ and $\left(Y_{t}, \mathscr{F}_{t}, P\right)_{t \in[0,1]}$ satisfy the hypothesis of Lemma 5. Let $v$ be the measure induced by the process $\left(Y_{t}, \mathscr{F}_{t}, P\right)$ on the measurable space 
$(\mathscr{C}, \mathscr{S})$. Then $v$ is mutually absolutely continuous with respect to $\mu$ and

$$
\frac{d \mu}{d v}(Y(\omega))=\exp \zeta(-\phi)(\omega)
$$

Remark. This corollary implies that the solutions of (22) are unique in a weak sense, i.e., all solutions of (22) which have continuous sample paths must induce the same measure on $(\mathscr{C}, \mathscr{S})$. The qualification "weak" is inserted because only the uniqueness of the probability law has been proved.

Recall that $\left(X_{t}, \mathscr{F}_{t}, P_{0}\right)_{t \in[0,1]}$ is an $F$-valued Brownian motion with continuous sample paths. Also recall Definition 8.

Definition 9. For any subset $\Lambda \subset \Phi$ define $\mathscr{D}(\Lambda) \subset L^{1}\left(\Omega, \mathscr{F}, P_{0}\right)$ by

$$
\mathscr{D}(\Lambda)=\{\exp \zeta(\phi) \mid \phi \in \Lambda\} \text {. }
$$

The corollary to Lemma 3 implies the next assertion.

Proposition 2. $\mathscr{D}\left(\Phi^{n}\right)$ is a bounded subset of $L^{2}\left(\Omega, \mathscr{F}, P_{0}\right)$.

LEMMA 6. $\mathscr{D}\left(\Phi^{n}\right)$ is a closed subset of $L^{2}\left(\Omega, \mathscr{F}, P_{0}\right)$.

Proof. Let $\phi_{1}, \phi_{2}, \cdots$ be a sequence from $\Phi^{n}$ and let $\rho$ be such that

$$
\lim _{n \rightarrow \infty} E_{0}\left|\rho-\exp \zeta\left(\phi_{n}\right)\right|^{2}=0
$$

and

$$
\lim _{n \rightarrow \infty} \exp \zeta\left(\phi_{n}\right)=\rho \quad \text { a.s. } P_{0} .
$$

Since $E_{0} \exp \zeta\left(\phi_{n}\right)=1$ for all $n, E_{0} \rho=1$, and so by Proposition 1 there is a functional $\psi$ such that $E_{0} \int_{0}^{1}|\psi(t, x)|_{H}^{2} d t<\infty$ and

$$
\rho=1+\int_{0}^{1}\left\langle\psi(t, X), d X_{s}\right\rangle \text { a.s. } P_{0} .
$$

Define the martingale $\rho_{t}$ by

$$
\rho_{t}=E_{0}\left\{\rho \mid \mathscr{F}_{t}\right\}, \quad t \in[0,1] .
$$

By taking modifications if necessary it can be assumed that the martingales $\rho_{t}$ and $\zeta^{t}\left(\phi_{n}\right)$ have continuous sample paths so that, by Doob's inequality [10, p. 353], it follows from (25) that

$$
\rho_{t}=\lim _{n \rightarrow \infty} \exp \zeta^{t}\left(\phi_{n}\right) \quad \text { uniformly on }[0,1] \text { a.s. } P_{0} .
$$

Next

$$
\exp \zeta\left(\phi_{n}\right)=1+\int_{0}^{1} \exp \zeta^{t}\left(\phi_{n}\right)\left\langle\phi_{n}(t), d X(t)\right\rangle
$$

so that from $(25),(26)$,

$$
\lim _{n \rightarrow \infty} E_{0} \int_{0}^{1}\left|\exp \zeta^{t}\left(\phi_{n}\right) \phi_{n}(t)-\psi(t)\right|_{H}^{2} d t=0,
$$

and hence by taking subsequences if necessary it can be assumed that

$$
\psi(t)=\lim _{n \rightarrow \infty} \exp \zeta^{t}\left(\phi_{n}\right) \phi_{n}(t) \quad \text { a.s. } l \otimes P_{0},
$$


where $l$ denotes Lebesgue measure on $[0,1]$. Now $\rho>$ a.s. $P_{0}$, because if $P_{0}(A)$ $=P_{0}\{\rho=0\}>0$, then (26), together with the fact that $\left|\phi_{n}\right|_{H} \leqq n$, implies that

$$
\lim _{n \rightarrow \infty} \int_{0}^{1}\left\langle\phi_{n}(t), d X(t)\right\rangle=-\infty \quad \text { on } A .
$$

But then

$$
\infty=E_{0} \int_{0}^{1}\left|\left\langle\phi_{n}(t), d X(t)\right\rangle\right|^{2} \leqq E_{0} \int_{0}^{1}\left|\phi_{n}(t)\right|_{H}^{2} d t \leqq N^{2},
$$

so that to avoid the contradiction one must have $\rho>0$ a.s. $P_{0}$. It follows that $\rho_{t}>0$ a.s., and hence combining (28) and (29) gives

$$
\frac{\psi(t)}{\rho(t)}=\lim _{n \rightarrow \infty} \phi_{n}(t) \quad \text { a.s. } l \otimes P_{0} .
$$

Thus there is a causal map $\phi \in \Phi^{n}$ such that

$$
\phi(t, X)=\lim _{n \rightarrow \infty} \phi_{n}(t, X) \quad \text { a.s. } l \otimes P_{0}
$$

and evidently $\rho=\exp \zeta(\phi)$.

The proofs of the next two results are identical respectively with the proofs of [2, Lemma 4] and [2, Thm. 2] with some obvious notational changes. Hence the proofs are omitted.

LEMMA 7. $\mathscr{D}\left(\Phi^{n}\right)$ is a convex subset of $L^{2}\left(\Omega, \mathscr{F}, P_{0}\right)$.

THEOREM 3. Let

$$
\mathscr{Y}^{0}=\left\{g \in \mathscr{Y} \mid E_{0} \exp \zeta(g)=1\right\} .
$$

Then $\mathscr{D}\left(\mathscr{Y}^{0}\right)$ is a closed, convex subset of $L^{1}\left(\Omega, \mathscr{F}, P_{0}\right)$.

5. Applications. The results developed above immediately imply the existence of optimal control laws for a broad class of problems. Consider the control system

$$
d X(t)=i f(t, X, u(t, X)) d t+d B(t), \quad t \in[0,1]
$$

with $X(0)=0$ a.s. Suppose that $f$ satisfies the assumptions $\mathrm{f} 1$ to $\mathrm{f} 5$ and in addition the function $f_{0}$ in $\mathrm{f} 4$ satisfies assumption $\mathrm{f6}$.

f6. There exists $K, K^{\prime}$ such that $f_{0}(n) \leqq K+K^{\prime} n$ for all $n \in R_{+}$.

Let $L: \mathscr{C} \rightarrow R$ be a fixed bounded $\mathscr{S}$-measurable function. For each $u \in \mathscr{U}$ the cost incurred by $u$ is defined to be

$$
J(u)=E_{0}\left[\left(\exp \zeta\left(g_{u}\right)\right) L(X)\right]=\int_{\Omega} L(X(\omega)) \exp \left(g_{u}(\omega)\right) d P_{0}
$$

THEOREM 4. Under assumptions f1 to $\mathrm{f} 6$, there exists an optimal control $u^{*} \in \mathscr{U}$, i.e.,

$$
J\left(u^{*}\right) \leqq J(u), \quad u \in \mathscr{U}
$$


Proof. From Lemma 3 it follows that $E_{0} \exp \zeta\left(g_{u}\right)=1$ for all $u \in \mathscr{U}$ and furthermore there exists $\alpha>1$ such that

$$
\sup _{u \in \mathscr{U}} E_{0} \exp \alpha \zeta\left(g_{u}\right)<\infty .
$$

Hence by [6, Chap. 2], $\mathscr{D}(\mathscr{Y})$ is a uniformly integrable subset of $L^{1}\left(\Omega, \mathscr{F}, P_{0}\right)$. By Theorem $3, \mathscr{D}(\mathscr{Y})$ is convex and strongly closed in $L^{1}\left(\Omega, \mathscr{F}, P_{0}\right)$. So that by [6, Chap. 2] $\mathscr{D}(\mathscr{Y})$ is weakly compact in $L^{1}\left(\Omega, \mathscr{F}, P_{0}\right)$. Hence the linear functional $\exp \zeta\left(g_{u}\right) \mapsto E_{0} L(X) \exp \zeta\left(g_{u}\right)$ attains a minimum.

In a manner corresponding exactly to the argument developed in [2], the results presented above can be used to obtain the existence of a saddle point for a class of two-person zero-sum games. Since there is nothing new here the details are omitted.

As an example of the class of the Brownian motions described here let $\left(B_{(t, \tau)}\right)_{(t, \tau) \in[0,1]^{2}}$ be a biadditive Gaussian process, i.e., a zero mean Gaussian process with independent increments in each coordinate of the index set such that

$$
E\left[B\left(t_{1}, \tau_{1}\right) B\left(t_{2}, \tau_{2}\right)\right]=\left(t_{1} \wedge t_{2}\right)\left(\tau_{1} \wedge \tau_{2}\right) .
$$

This stochastic process has continuous sample paths and when it is considered as indexed by one coordinate of the index set, it is a Brownian motion with values in the Banach space of continuous functions, $C[0.1]$. Optimal control results can be obtained by the previous results for stochastic differential equations with this Brownian motion.

Remark on [2]. It is necessary to make the assumption of uniform integrability of the family of densities to obtain the results in Theorems 4 and 5 of [2]. One sufficient condition for this uniform integrability is that the growth of the drift term is at most linear. This fact is shown by Beneš [1] by verifying that the family of densities have a bounded $\alpha$ th moment for some $\alpha>1$.

\section{REFERENCES}

[1] V. E. BENEŠ, Existence of optimal stochastic control laws, this Journal, 9 (1971), pp. 446472.

[2] T. DunCan AND P. VARAIYA, On the solutions of a stochastic control system, this Journal, 9 (1971), pp. $354-371$.

[3] R. M. Dudley, J. Feldman ANd L. LeCam, On seminorms and probabilities, and abstract Wiener spaces, Ann. of Math., 93 (1971), pp. 390-408.

[4] L. Gross, Abstract Wiener spaces, Proc. Fifth Berkeley Symposium on Math. Stat. and Prob., University of California Press, Berkeley, Calif., 1965.

[5] I. E. Segal, Distributions in Hilbert space and canonical systems of operators, Trans. Amer. Math. Soc., 88 (1958), pp. 12-41.

[6] P. A. Meyer, Probability and Potentials, Blaisdell, Waltham, Mass., 1966.

[7] X. FerniQue, Integrabilité des vecteurs gaussians, C.R. Acad. Sci. Paris Sér. A-B, 270 (1970), pp. 1698-1699.

[8] E. Nelson, An existence theorem for second order parabolic equations, Trans. Amer. Math. Soc., 88 (1958), pp. $414-429$.

[9] L. Gross, Potential theory on Hilbert space, J. Functional Analysis, 1 (1967), pp. 123-181.

[10] J. L. Doob, Stochastic Processes, John Wiley, New York, 1953.

[11] K. Itô, Multiple Wiener integral, J. Math. Soc. Japan, 3 (1951), pp. 157-169.

[12] N. WiEnER, The homogeneous chaos, Amer. J. Math., 60 (1938), pp. 897-936.

[13] R. H. CAMERON AND W. T. MARTIN, Transformation of Wiener integrals under a general class of linear transformations, Trans. Amer. Math. Soc., 58 (1945), pp. 184-219.

[14] I. E. Segal, Tensor algebras over Hilbert space I, Ibid., 81 (1956), pp. 106-134. 
[15] H. Kunita ANd S. Watanabe, On square integrable martingales, Nagoya Math. J., 30 (1967), pp. 209-245.

[16] I. V. GiRSANOv, On transforming a certain class of stochastic processes by absolutely continuous substitution of measures, Theor. Probability Appl., 5 (1960), pp. 285-301.

[17] T. E. Duncan, Transforming Fréchet-valued Brownian motion by absolute continuity of measures, unpublished.

[18] A. Bensoussan, Généralization du théorème de Girsanov, to appear.

[19] C. DolÉAns-DADE, Quelques applications de la formule de changement de variables pour les semimartingales, Z. Wahrscheinlichkeitstheorie und Verw. Gebiete, 16 (1970), pp. 181-194.

[20] M. LoÉve, Probability Theory, 2nd ed., Van Nostrand, Princeton, N.J., 1960.

[21] V. E. BENE, Existence of optimal strategies based on specified information for a class of stochastic decision problems, this Journal, 8 (1970), pp. 179-188. 